\title{
Zoneamento da área extrativista mais produtiva da Anomalocardia flexuosa (Linnaeus, 1767) (Mollusca: Bivalvia) no mundo
}

\author{
Severino Adriano de Oliveira Lima ${ }^{1 *}$ \\ Humber Agrelli Andrade ${ }^{2}$ \\ ${ }^{1}$ Universidade Federal de Rondônia, Departamento de Engenharia de Pesca \\ Rua da Paz, 4376, Lino Alves Teixeira, Presidente Médici - RO, Brasil \\ ${ }^{2}$ Universidade Federal Rural de Pernambuco, Departamento de Pesca e Aquicultura \\ Rua Dom Manoel de Medeiros, s/n, CEP 52171-900, Recife - PE, Brasil \\ * Autor para correspondência \\ dianooliveira@hotmail.com
}

Submetido em 23/03/2018

Aceito para publicação em 31/07/2018

\section{Resumo}

A praia de Mangue Seco, no estado de Pernambuco, é um local com grande quantidade de ecossistemas e se destaca principalmente pela captura da espécie Anomalocardia flexuosa. Neste trabalho foi proposto um zoneamento dessa praia de acordo com as características mais expressivas e também considerando as densidades da Anomalocardia flexuosa. No delineamento da região foram considerados primeiramente os efeitos das águas circundantes e a proximidade da linha de praia; assim, três principais estratos de localização foram avaliados (borda, interior costa e interior mar), além de estratos secundários com divisões latitudinais e longitudinais. No total foram obtidas 176 amostras em campanhas realizadas no ano de 2015. As médias das densidades foram maiores no setor interior costa em todas as campanhas. As zonas localizadas próximas a estuários pareceram mais propícias ao estabelecimento da espécie quando comparadas a locais com maior interferência marinha. Foi possível diferenciar, principalmente, quatro estratos de localização de acordo com as características morfodinâmicas e as densidades da Anomalocardia flexuosa. O primeiro estrato e o mais abundante seria o interior costa, o segundo o interior mar e o estrato borda seria dividido em dois substratos, um mais afastado da linha de praia e outro mais próximo às saídas de foz.

Palavras-chave: Anomalocardia brasiliana; Densidade; Distribuição; Ecossistema costeiro; Macrofauna

\section{Abstract}

Zoning of the most productive extraction area of Anomalocardia flexuosa (Linnaeus, 1767) (Mollusca: Bivalvia) in the world. Mangue Seco Beach, in Pernambuco State, is a region with a large number of ecosystems and is notable as a place where Anomalocardia flexuosa is collected. In this work, zoning for the beach is proposed based on to the most expressive characteristics of the region and densities of $A$. flexuosa. In the delineation of the region, the effects of the surrounding water and proximity to the beach line were considered first. Three main location strata were evaluated, border, interior coast and interior sea, as well as secondary strata with latitudinal and longitudinal divisions. In total, 176 samples were obtained during surveys carried out in 2015. For all surveys the mean densities were higher in the interior coastal sector. The areas located near estuaries 
seemed better for the species compared to regions with greater marine interference. In general, it was possible to differentiate four location strata according to the morphodynamic characteristics and densities of $A$. flexuosa. The first and most abundant stratum would be the interior coast, the second stratum would be the interior sea and the border stratum would be divided into two substrates, one further away from the beach line and another closer to the river mouth.

Key words: Anomalocardia brasiliana; Coastal Ecosystem; Density; Distribution; Macrofauna

\section{Introdução}

A espécie Anomalocardia fleuxuosa é um recurso importante para vários estados litorâneos do Brasil. Ressalta-se que a espécie tem sinonímia de Anomalocardia brasiliana, porém a plataforma especializada em taxonomia WORMS (World Register of Marine Species) reconhece a designação A. flexuosa (MOLlUSCABASE, 2018). Além disso, no Brasil existem vários nomes locais para a espécie, sendo marisco em Pernambuco, massunim em vários outros estados do Nordeste e berbigão nos estados do Sul e Sudeste.

A captura de $A$. flexuosa é realizada em praticamente todos os estados litorâneos do Brasil, com grande importância econômica e social (SILVA-CAVALCANTI; COSTA, 2011). Em Pernambuco a importância deste molusco bivalve é ainda mais acentuada, pois, até meados da década de 2000, a A. flexuosa foi a espécie com maior produção entre todos os recursos pesqueiros, com aproximadamente 4716 t extraídas no ano de 2007, de acordo com dados disponíveis para consulta pública e irrestrita sobre a pesca no Brasil (IBAMA, 2009). As produções nos demais estados do Brasil são bem menores, com a Paraíba ocupando a segunda posição com uma captura de 1358 t em 2007, o que correspondeu a aproximadamente um terço da produção de Pernambuco. Não há informações estatísticas para períodos recentes. A principal área de extração da $A$. flexuosa em Pernambuco é localizada no município de Igarassu, que tem como único banco extrativista a praia de Mangue Seco, sendo que aproximadamente 50\% da produção pernambucana advém desse banco (IBAMA/ CEPENE, 2008). A atividade continua intensa desde os últimos registros estatísticos e provavelmente Mangue Seco continua sendo o principal ponto de extração mundial da espécie.
A praia de Mangue Seco é um local com grande influência estuarina, assim, o fluxo da maré é um dos fatores mais importante para atividade pesqueira (LESSA et al., 2011) e que influi também diretamente na distribuição da $A$. flexuosa. Por causa da presença de extensos estuários, além de uma grande quantidade de ecossistemas altamente produtivos, em que se encontram áreas cobertas de coqueiros, recifes e uma ilhota, a praia é considerada uma "região verde" (OLIVEIRA et al., 2011). A zona intertidal em algumas áreas pode ter mais de 2000 metros no sentido oesteleste e diferenças morfológicas internas são evidentes (OLIVEIRA, observação pessoal). Essas diferenças são mais perceptíveis nos extremos que têm aporte fluvial nos sentidos norte e sul e marinho no sentido Leste. Na porção extrema ao norte do banco fica localizada a foz do Canal de Santa Cruz, que é o maior complexo estuarino de Pernambuco (MOURA, 2009) e tem grande atividade pesqueira (IBAMA/CEPENE, 2008). Já o extremo sul fica perto da foz do rio Timbó, que também tem prática intensa de pesca (CABRAL et al., 2006). Dadas as diferenças perceptíveis internamente em Mangue Seco, os trabalhos de El-Deir et al. (2009) e Oliveira et al. (2011) propuseram a divisão territorial para avaliar as densidades da A. flexuosa no local, nos sentidos latitudinal e longitudinal, porém em ambos os trabalhos a totalidade da área não foi contemplada.

Dispor de informações em partes de um mesmo local permite comparações e análises que são úteis para a gestão do local. No Brasil a Política Nacional do Meio Ambiente (PNMA) instituiu o zoneamento ambiental (Lei ํㅡ 6.938/1981) como um instrumento para contribuir na preservação, melhoria e recuperação da qualidade ambiental, bem como no desenvolvimento socioeconômico, na segurança nacional e na proteção à dignidade da vida humana. Em 2002 esse instrumento passou a ser chamado de zoneamento ecológico- 
econômico (ZEE), regulamentado pelo decreto no 4.297/2002. Os trabalhos sobre ZEE no Brasil são elaborados por diferentes métodos e objetivos sem seguir um padrão, porém os aspectos ambientais são sempre utilizados para estratificação do local (SANTOS; RANIERI, 2013).

Um planejamento adequado para o zoneamento territorial requer a necessidade de se conhecer os recursos disponíveis na região em termos quantitativos (SANTOS; RANIERI, 2013). Na praia de Mangue Seco o principal recurso vem da extração da A. flexuosa, que tem densidades elevadas na área (OLIVEIRA et al., 2011). Portanto, neste trabalho foi proposto um zoneamento da praia de Mangue Seco de acordo com as características mais expressivas dessa área e também considerando as densidades do principal recurso explorado na região, que é o bivalve $A$. flexuosa, assim contribuindo com a gestão da atividade extrativista da região.

\section{Material e Métodos}

\section{Área de estudo e zoneamento territorial}

A praia de Mangue Seco fica no município de Igarassu que faz parte da Região Metropolitana do Recife. A planície se estende no sentido sul-norte, desde a foz do Rio Timbó até a foz sul do Canal de Santa Cruz, abrangendo uma área de aproximadamente 2,7 $\mathrm{km}^{2}$ (Figura 1). A linha de praia tem aproximadamente $2 \mathrm{~km}$ de extensão, com águas rasas e pequenas ondas (OLIVEIRA et al., 2014).

No zoneamento inicial proposto para a região, foram considerados primeiramente os efeitos das águas circundantes e a proximidade da linha de praia, assim três principais estratos de localização foram observados e denominados de borda (b1, b2, b3 e b4), interior costa (ic) e interior mar (im) (Figura 1A). O

FIGURA 1: Praia de Mangue Seco localizada no município de Igarassu (PE). Painel superior indica os estratos de localização borda (b1, b2, b3 e b4), interior costa (ic) e interior mar (im) (A). Painéis centrais indicam a subdivisão latitudinal em quatro campanhas realizadas em julho (B), agosto $(\mathrm{C})$, setembro (D) e novembro (E). Painéis inferiores indicam a subdivisão longitudinal em quatro campanhas realizadas em julho (B), agosto (C), setembro (D) e novembro (E). Os pontos dos mapas são as coordenadas em que as coletas foram realizadas, sendo: borda (triângulos abertos), interior costa (círculos fechados) e interior mar (quadrados abertos).

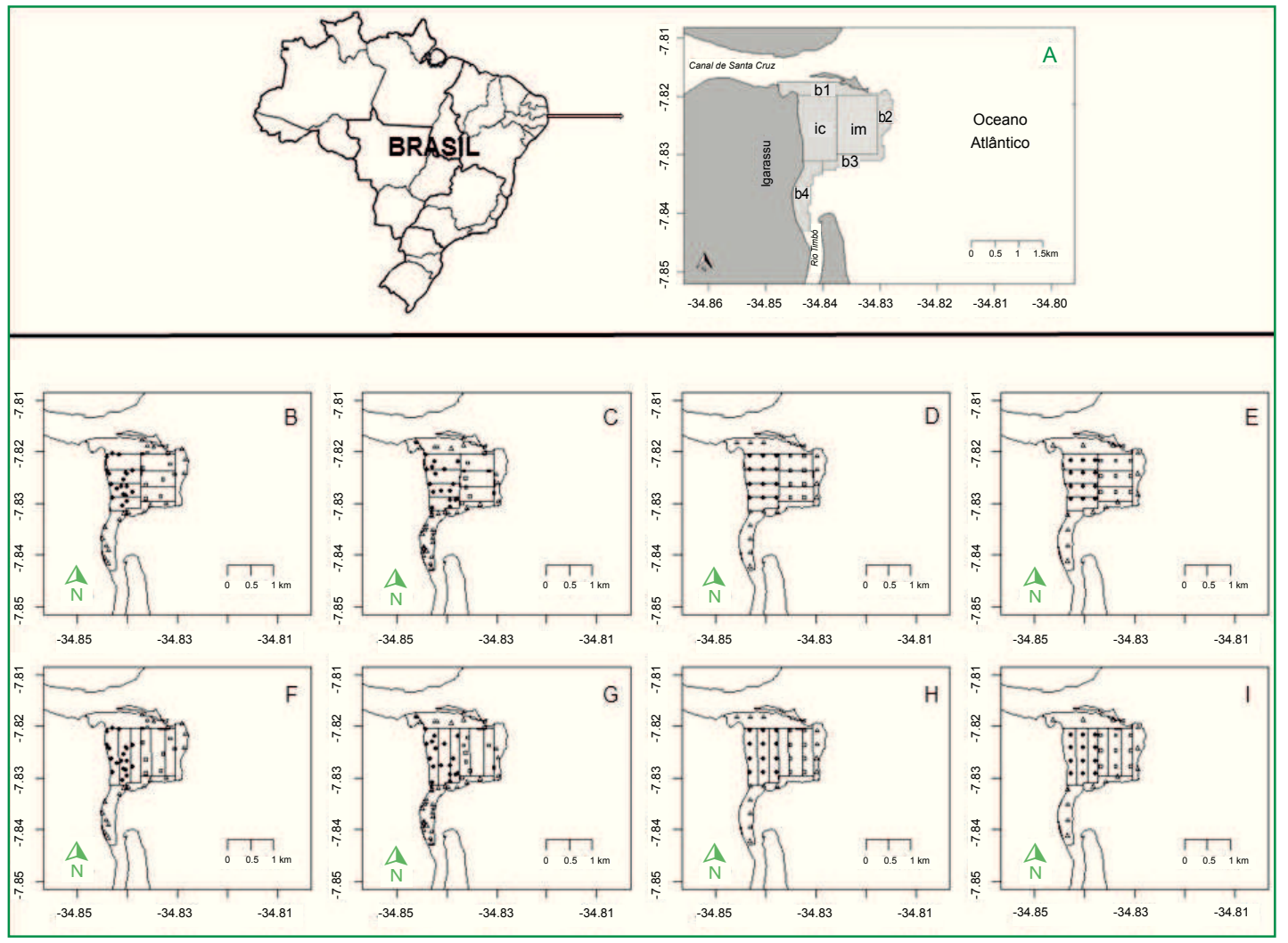


primeiro corresponde à região de extremos do banco, mais exposta à ação da água circundante; supõe-se que nessa área ocorra maior interferência de ação de ondas e correntes. O segundo estrato é uma região mais interior e próxima da linha de praia, que parece propiciar uma zona mais abrigada e menos exposta a ondas e correntezas. A terceira localização está também no interior do banco, porém mais intermediária entre à linha da praia e o mar. Subgrupos de localização dos estratos principais também foram avaliados. A borda foi dividida em quatro áreas, estando a borda 1 (b1) localizada mais ao norte do banco e próximo a foz do Canal de Santa Cruz, a borda 2 (b2) localizada na região mais externa no sentido Oeste-leste com moderado ou sem aporte fluvial, a borda 3 (b3) situada entre a foz do rio Timbó e a região mais externa e também tem moderado aporte fluvial e a borda 4 (b4) fica na porção mais ao sul do banco e perto da foz do rio Timbó. Os interiores costa e mar foram divididos de duas maneiras distintas, a primeira no sentido latitudinal em que as subáreas no interior costa foram ordenadas de um a quatro no sentido norte-sul e de um a três no interior mar no mesmo sentido (Figura 1 - painéis centrais). A segunda divisão dos estratos do interior foi no sentido longitudinal e ambas (interior costa e interior mar) ordenadas de um a três no sentido oeste-leste (Figura 1 - painéis inferiores).

\section{Coleta dos dados}

No total foram obtidas 176 amostras em quatro campanhas realizadas no ano de 2015, sendo 47 no mês de julho (Figura $1 \mathrm{~B}$ e F), 61 em agosto (Figura $1 \mathrm{C}$ e G), 34 em setembro (Figura $1 \mathrm{D}$ e H) e 34 em novembro (Figura $1 \mathrm{E} \mathrm{e} \mathrm{I).} \mathrm{As} \mathrm{coletas} \mathrm{dos} \mathrm{meses} \mathrm{de}$ julho e agosto foram realizadas aleatoriamente ao longo de todo o banco. Nos meses de setembro e novembro as coletas foram sistemáticas, com 34 pontos, sendo que no primeiro foram colocadas de maneira equidistante ao longo de todo o banco e no segundo também, porém considerando cada estrato de localização. A quantidade de amostras realizadas por campanha e nos estratos de localização é mostrada na Tabela 1. As amostras foram

TABELA 1: Número de amostras coletadas nos estratos de localização no banco da praia de Mangue Seco.

\begin{tabular}{lcccccc}
\hline Configuração dos estratos & Estrato de localização & Julho & Agosto & $\begin{array}{c}\text { Amostras } \\
\text { Setembro }\end{array}$ & Novembro & Total \\
\hline \multirow{2}{*}{$\begin{array}{l}\text { Estratos de localização } \\
\text { primários }\end{array}$} & Borda & 21 & 37 & 14 & 13 & 85 \\
& Interior Costa & 18 & 17 & 12 & 12 & 59 \\
& Interior Mar & 7 & 7 & 8 & 9 & 31 \\
\hline \multirow{4}{*}{ Estratos de localização } & Borda 1 & 7 & 8 & 3 & 3 & 21 \\
secundários & Borda 2 & 3 & 3 & 4 & 3 & 13 \\
& Borda 3 & 3 & 5 & 1 & 3 & 12 \\
& Borda 4 & 8 & 21 & 6 & 4 & 39 \\
\hline \multirow{3}{*}{ Configuração latitudinal } & Interior Costa 1 & 2 & 6 & 3 & 3 & 14 \\
& Interior Costa 2 & 6 & 1 & 3 & 3 & 13 \\
& Interior Costa 3 & 7 & 5 & 3 & 3 & 18 \\
& Interior Costa 4 & 3 & 5 & 3 & 3 & 14 \\
& Interior Mar 1 & 2 & 1 & 4 & 3 & 10 \\
& Interior Mar 2 & 3 & 3 & 2 & 3 & 11 \\
& Interior Mar 3 & 2 & 3 & 2 & 3 & 10 \\
\hline \multirow{5}{*}{ Configuração longitudinal } & Interior Costa 1 & 6 & 8 & 4 & 4 & 22 \\
& Interior Costa 2 & 10 & 3 & 4 & 4 & 21 \\
& Interior Costa 3 & 2 & 6 & 4 & 4 & 16 \\
& Interior Mar 1 & 2 & 5 & 0 & 3 & 14 \\
& Interior Mar 2 & 3 & 0 & 4 & 3 & 6 \\
& Interior Mar 3 & 2 & 2 & 4 & 3 & 11 \\
\hline
\end{tabular}


realizadas com um coletor cilíndrico de policloreto de polivinila (PVC) de $19 \mathrm{~cm}$ de diâmetro que foi introduzido no sedimento a $10 \mathrm{~cm}$ de profundidade. O sedimento, junto com os organismos, foi lavado com água e peneirado com malha quadrada de $1 \mathrm{~mm}$ entre nós adjacentes e os organismos foram contabilizados posteriormente em laboratório.

\section{Análise dos dados}

Nas análises das densidades da A. flexuosa em Mangue Seco foram utilizados métodos geoestatísticos com interpolações calculadas com o inverso da distância para inspeções visuais, usando o pacote gstat no $\mathrm{R}$ (PEBESMA; GRAELER, 2017). Sumários estatísticos básicos com valores das médias, máximos e mínimos das densidades nos estratos de localizações foram calculados. Pressupostos de normalidade e homocedasticidade não foram atendidos e provas não paramétricas de Kruskal-Wallis (KRUSKAL; WALLIS, 1952) foram alternativas para avaliar diferenças significativas $(\alpha=0,05)$ das densidades nos estratos de localização.
Nos casos de diferenças observadas procedeu-se com teste de comparações múltiplas. Para todas as análises foi utilizado o programa R (R CORE TEAM, 2017).

\section{Resultados}

As médias das densidades de $A$. flexuosa tiveram valores maiores no setor ic do banco em todas as campanhas realizadas (Figura 2). Nas amostras de julho (Figura 2 A) as densidades médias foram de 571 ind. $\mathrm{m}^{-2}$, 914 ind. $\mathrm{m}^{-2}$ e 262 ind. $\mathrm{m}^{-2}$ para os estratos, borda, ic e im, respectivamente, e agregações com máximas densidades foram encontradas na parte mais central do ic (3599 ind. $\mathrm{m}^{2}$ ) e na região mais próxima à foz do rio Timbó da borda (2541 ind. $\mathrm{m}^{2}$ ). $\mathrm{O}$ mês de agosto (Figura $2 \mathrm{~B}$ ) teve médias de densidades de 263 ind. $\mathrm{m}^{-2}, 1013$ ind. $\mathrm{m}^{-2}$ e 242 ind. $\mathrm{m}^{-2}$ para a borda, ic e im, respectivamente, e o ponto com maior densidade (4058 ind. $\mathrm{m}^{2}$ ) para todas as campanhas localizado no estrato ic. No mês de setembro (Figura 2 C) as densidades médias para a borda, ic e im foram de 267 ind. $\mathrm{m}^{-2}, 938$ ind. $\mathrm{m}^{-2}$ e 234 ind. $\mathrm{m}^{-2}$, respectivamente, $\mathrm{e}$

FIGURA 2: Interpolações das densidades da Anomalocardia flexuosa na praia de Mangue Seco nas campanhas de julho (A), agosto (B), setembro (C) e novembro (D). Triângulos abertos, círculos fechados e quadrados abertos representam amostras nos estratos de localização de borda, interior costa e interior mar, respectivamente.

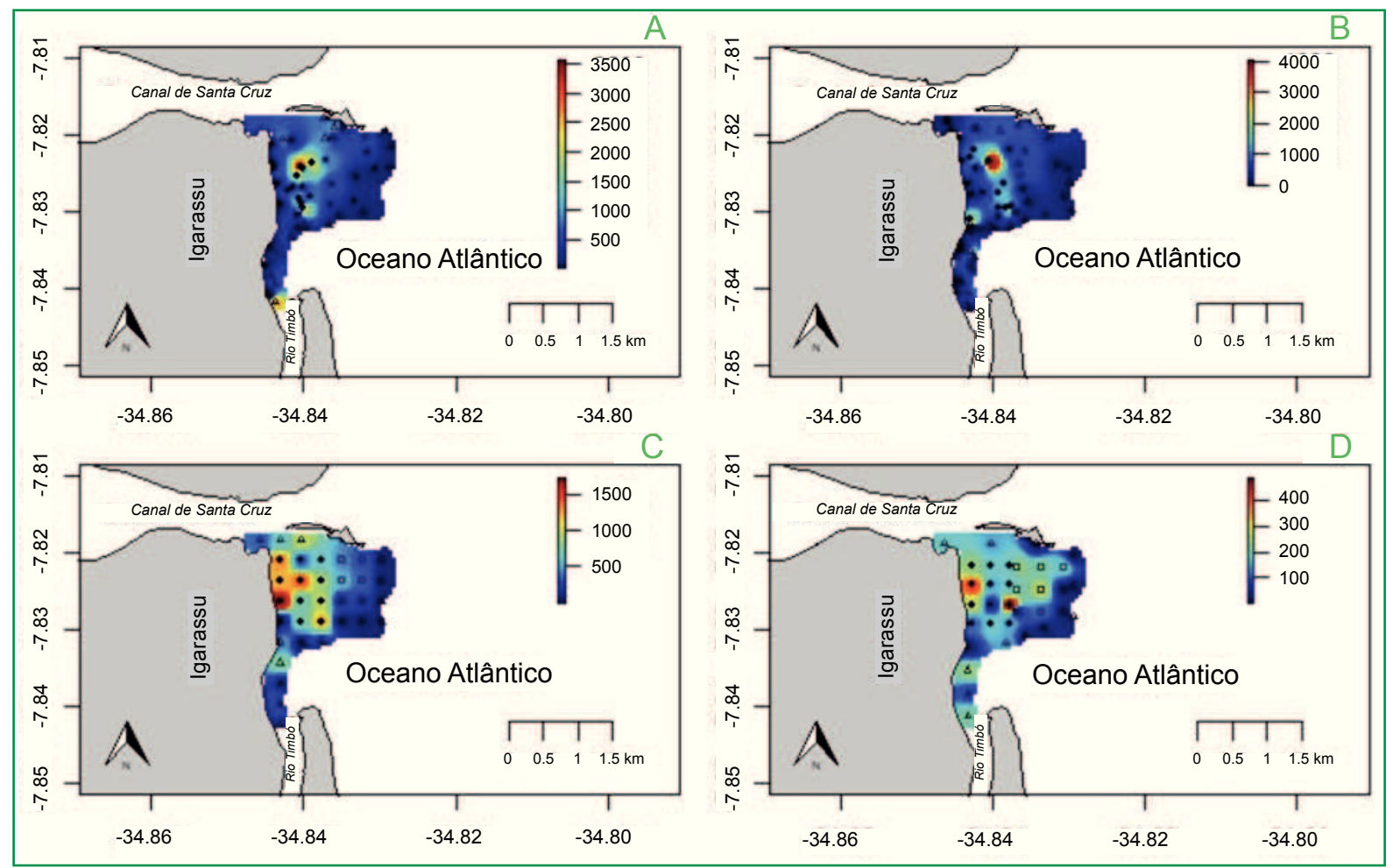


agregações com densidades elevadas foram encontradas na maioria dos pontos da região ic com máximo de 1729 ind. $\mathrm{m}^{2}$ e na borda próxima ao Canal de Santa Cruz com 988 ind. $\mathrm{m}^{2}$. Em novembro (Figura $2 \mathrm{D}$ ) as densidades médias para borda (76 ind. $\left.\mathrm{m}^{-2}\right)$, ic $\left(215\right.$ ind. $\left.\mathrm{m}^{-2}\right)$ e im (141 ind. $\mathrm{m}^{-2}$ ) foram as menores entre todas as campanhas realizadas, e a agregação com densidade máxima ocorreu no estrato ic (494 ind. $\mathrm{m}^{2}$ ). Nos subgrupos da borda, as densidades médias de b1 e b4 sempre foram maiores do que em b2 e b3 para todas as campanhas. A menor média da densidade ( 0 ind. $\left.\mathrm{m}^{-2}\right)$ ocorreu em b2 no mês de novembro e a maior em b4 (665 ind. $\left.\mathrm{m}^{-2}\right)$ em julho. As médias das densidades em Mangue Seco neste trabalho são maiores do que a maioria dos bancos estudados em outros locais do Brasil (Tabela 2).

As medianas das densidades de $A$. flexuosa foram comparadas nos estratos de localização primários para cada campanha e, de maneira geral, o estrato interior costa (ic) foi o que mais se destacou (Figura 3). Diferenças significativas foram observadas em todas as campanhas

TABELA 2: Informações das densidades médias da Anomalocardia flexuosa publicadas em outros locais do Brasil e também na praia de Mangue Seco em anos anteriores.

\begin{tabular}{cccccc}
\hline Estado & Local & Latitude & Ano da coleta & ind.m ${ }^{-2}$ & Fonte \\
\hline Rio Grande do Norte & Praia de Pernambuquinho & $04^{\circ} 55^{\prime} \mathrm{S}$ & $2007-2008$ & $100-1148$ & Rodrigues et al. (2013) \\
Rio Grande do Norte & Praia da Barra & $04^{\circ} 55^{\prime} \mathrm{S}$ & $2008-2008$ & $951-1813$ & Rodrigues et al. (2013) \\
Pernambuco & Estuário de Goiana & $0^{\circ} 55^{\prime} \mathrm{S}$ & $2009-2010$ & $284-706$ & Silva-Cavalcanti et al. (2018) \\
Pernambuco & Praia de Mangue Seco & $0^{\circ} 83^{\circ} \mathrm{S}$ & $2006-2007$ & $816-2016$ & El-Deir et al. (2009) \\
Pernambuco & Praia de Mangue Seco & $0^{\circ} 87^{\circ} \mathrm{S}$ & 2009 & $103-415$ & Oliveira et al. (2011) \\
Pernambuco & Praia de Mangue Seco & $0^{\circ} 83^{\circ} \mathrm{S}$ & 2015 & $0-1013$ & Este estudo \\
Rio de Janeiro & Praia de Flexeiras & $22^{\circ} 56^{\prime} \mathrm{S}$ & $2006-2009$ & 289 & Mattos e Cardoso (2012) \\
São Paulo & Praia Cidade & $23^{\circ} 37^{\prime} \mathrm{S}$ & $2007-2008$ & 53 & Corte et al. (2015) \\
São Paulo & Baia do Araçá & $23^{\circ} 49^{\prime} \mathrm{S}$ & $2008-2009$ & 52 & Corte et al. (2017) \\
Paraná & Baia de Paranaguá & $25^{\circ} 30^{\prime} \mathrm{S}$ & $1996-1997$ & $95-489$ & Boehs et al. (2008) \\
\hline
\end{tabular}

FIGURA 3: Diagramas de caixas das densidades de Anomalocardia flexuosa nas posições borda, interior costa (ic) e interior mar (im) na praia de Mangue Seco nas campanhas de julho (A), agosto (B), setembro (C) e novembro (D). As letras minúsculas nos painéis indicam os resultados dos testes de hipótese.

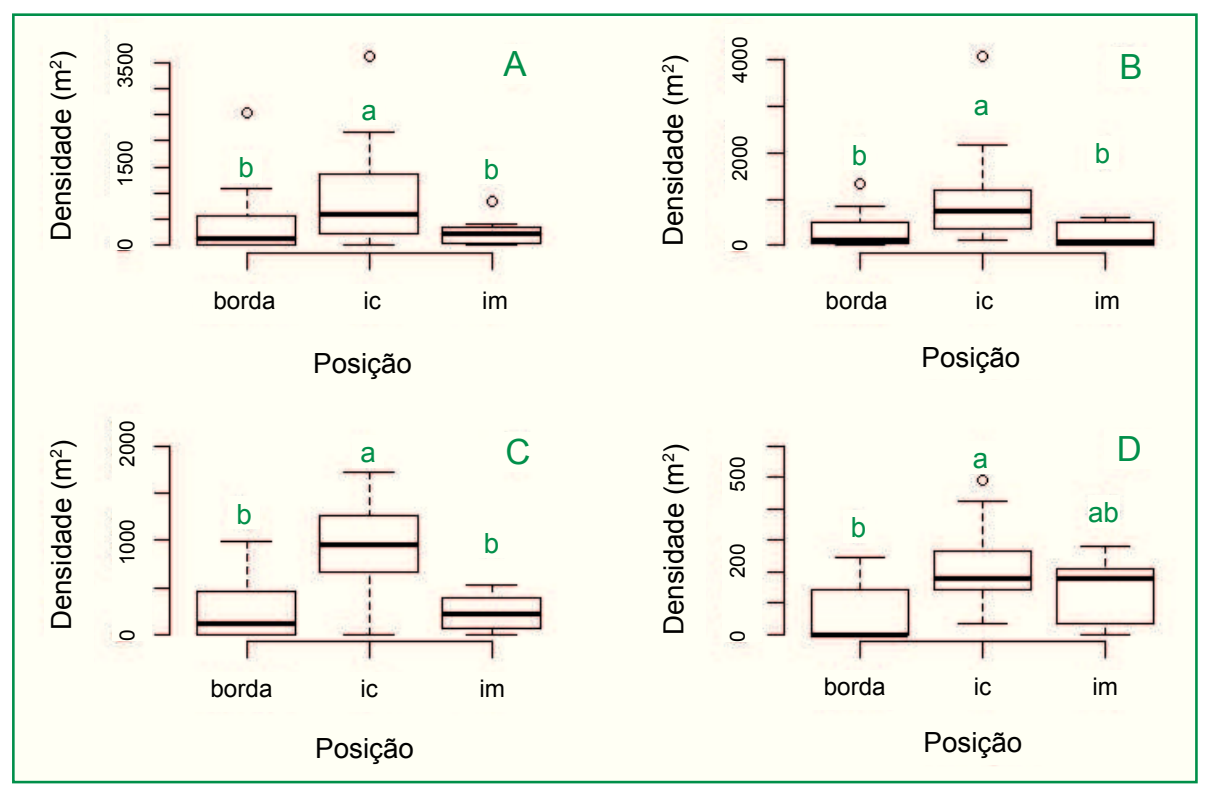


$(p<0,05)$. Nos testes de comparações múltiplas, a densidade em ic (582 ind. $\mathrm{m}^{-2}$ ) foi significativamente maior do que na borda (141 ind. $\mathrm{m}^{-2}$ ) e no im (212 ind. $\mathrm{m}^{-2}$ ) em julho (Figura $3 \mathrm{~A}$ ). A mesma diferença significativa ocorreu em agosto (Figura $3 \mathrm{~B}$ ), com valores de mediana de 741 ind. $\mathrm{m}^{-2}, 106$ ind. $\mathrm{m}^{-2}$ e 71 ind. $\mathrm{m}^{-2}$ para ic, borda e im, respectivamente, e também em setembro (Figura $3 \mathrm{C}$ ), com medianas de 953 ind. $\mathrm{m}^{-2}, 123$ ind. $\mathrm{m}^{-2}$ e 211 ind. $\mathrm{m}^{-2}$ para ic, borda e im, respectivamente. No mês de novembro (Figura 3 D), a densidade em ic (176 ind. $\mathrm{m}^{-2}$ ) foi significativamente maior do que na borda $\left(0\right.$ ind. $\left.\mathrm{m}^{-2}\right)$, mas não do que no im $\left(176\right.$ ind. $\left.\mathrm{m}^{-2}\right)$.

As medianas das densidades de A. flexuosa foram comparadas nos estratos de localização secundários com todas as campanhas juntas (Figura 4). As densidades apresentaram diferença significativa na configuração latitudinal e também longitudinal $(p<0,05)$. No sentido latitudinal (Figura 4 A) o teste de comparações múltiplas apontou que as densidades nos subgrupos ic1 (353 ind. $\left.\mathrm{m}^{-2}\right)$, ic2 (917 ind. $\left.\mathrm{m}^{-2}\right)$, ic3 (547 ind. $\left.\mathrm{m}^{-2}\right)$, ic4 $\left(547\right.$ ind. $\left.\mathrm{m}^{-2}\right)$ e im1 (318 ind. $\left.\mathrm{m}^{-2}\right)$ são significativamente maiores do que nos subgrupos b2 $\left(0\right.$ ind. $\left.\mathrm{m}^{-2}\right)$, b3 (35 ind. $\left.\mathrm{m}^{-2}\right)$, im2 (141 ind. $\left.\mathrm{m}^{-2}\right)$ e im3 (18 ind. $\left.\mathrm{m}^{-2}\right)$. No sentido longitudinal (Figura 4 B) o teste de comparações múltiplas distinguiu que as densidades nos subgrupos ic1 (300 ind. $\left.\mathrm{m}^{-2}\right)$, ic2 (494 ind. $\left.\mathrm{m}^{-2}\right)$ e ic3 (47 ind. $\left.\mathrm{m}^{-2}\right)$ são significativamente maiores do que nos subgrupos b2 $\left(0\right.$ ind. $\left.\mathrm{m}^{-2}\right), \mathrm{b} 3\left(35\right.$ ind. $\left.\mathrm{m}^{-2}\right), \mathrm{im} 2\left(141\right.$ ind. $\left.\mathrm{m}^{-2}\right)$ e im 3 $\left(0\right.$ ind. $\left.\mathrm{m}^{-2}\right)$.

\section{Discussão}

$\mathrm{Na}$ divisão primária proposta no banco da praia de Mangue Seco ficou evidente que o estrato ic é mais propício para as agregações da espécie quando comparado aos outros dois estratos primários (borda e im). Por ser um estrato aparentemente mais abrigado e protegido das ações de ondas em relação à borda e ao im, a proliferação da A. flexuosa é facilitada, pois locais com pouco material em suspensão são os ideais para o estabelecimento da espécie, que tem sifões curtos e fundidos, com tentáculos simples, o que impede maiores agregações em áreas com ressuspensão frequente de sedimentos (NARCHI, 1974), como deve acontecer na borda e no im, que são locais mais próximos aos efeitos das águas circundantes. A variabilidade no setor ic também foi mais alta, o que denota que estudos no banco devem ter um esforço amostral maior nesse estrato.

Nas divisões secundárias as regiões b1 e b4 parecem mais benéficas para $A$. flexuosa do que as b2 e b3. As características físicas e morfodinâmicas de b1 e b4 são parecidas, pois são áreas próximas à saída de estuários que reconhecidamente contribuem com

FIGURA 4: Diagramas de caixas das densidades de Anomalocardia flexuosa nos estratos de localização secundários na praia de Mangue Seco. (A) Divisão latitudinal nas posições de borda 1, 2, 3 e 4 (b1, b2, b3, b4), na posição interior costa 1, 2, 3 e 4 (ic1, ic2, ic3, ic4) e no interior mar (im1, im2, im3). (B) Divisão longitudinal nas posições de borda 1, 2, 3 e 4 (b1, b2, b3, b4), na posição interior costa 1, 2 e 3 (ic1, ic2, ic3) e no interior mar (im1, im2, im3). As letras minúsculas nos painéis indicam os resultados dos testes de hipótese.

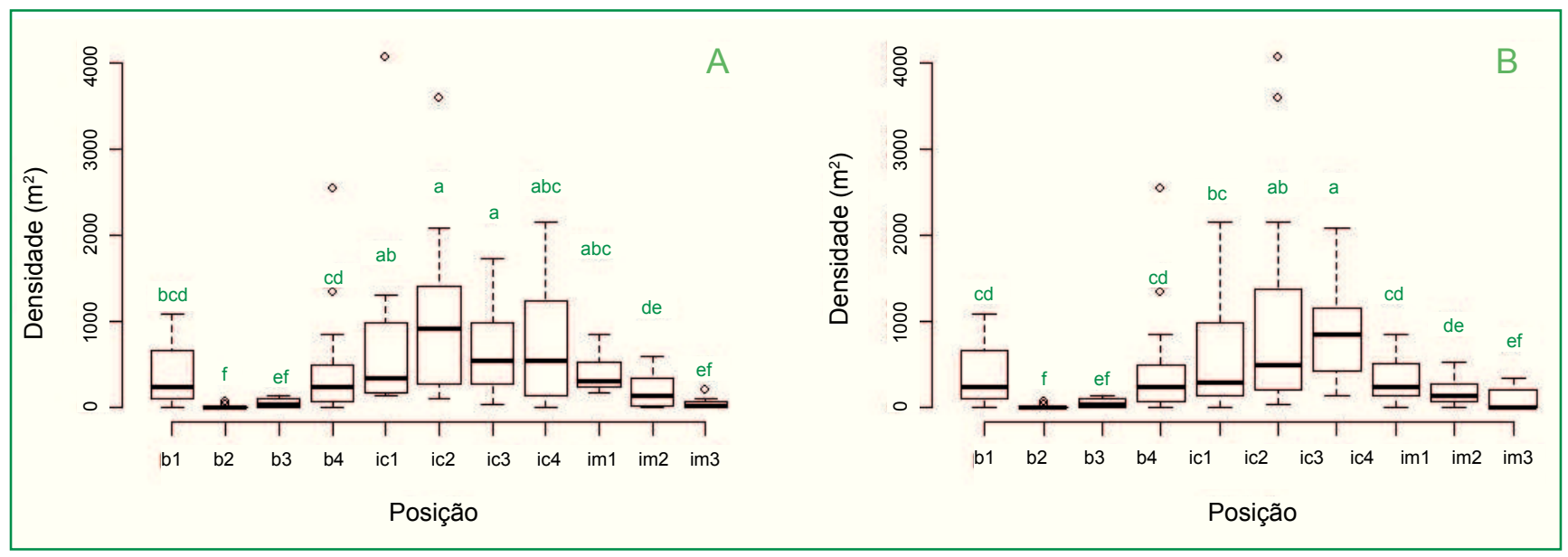


grande aporte de sedimentos finos, que são os mais adequados para a espécie (NARCHI, 1974), e também de matéria orgânica, que é um dos parâmetros abióticos que mais interferem na distribuição do bivalve, que tem preferência por áreas com alto teor de matéria orgânica (BOEHS et al., 2008; BELÉM et al., 2013), enquanto b2 e b3 são áreas com maior ação de ondas.

Avaliações espaciais são corriqueiras em estudos de distribuição da $A$. flexuosa inclusive para Mangue Seco (e.g. EL-DEIR et al., 2009; OLIVEIRA et al., 2011), porém de maneira geral procura-se contemplar a totalidade da área envolvida. A distribuição da A. flexuosa no banco de Mangue Seco, de acordo com os resultados deste trabalho, foi mais ampla do que os delineamentos amostrais propostos por El-Deir et al. (2009) e Oliveira et al. (2011). No primeiro, foram apenas consideradas localizações próximas à saída do Canal de Santa Cruz, Coroa do Avião e locais próximos à linha de praia, desconsiderando a região próxima ao Rio Timbó e mais externas do banco no sentido do mar (leste), que corresponderia aos estratos im, b2, b3 e b4. No segundo, os autores propuseram uma divisão longitudinal e latitudinal, porém os extremos do banco no sentido mar (Leste) não foram apreciados o que corresponderiam aos estratos im, b1, b2 e b3. Os delineamentos dos referidos trabalhos apesar de informativos são apenas restritos a regiões que apresentam aparentemente densidades mais elevadas, porém na divisão do banco proposta neste trabalho várias feições das distribuições em termos de densidades da espécie podem ser verificadas.

A distribuição da A. flexuosa também foi estudada em outros locais do Brasil. Em uma praia do estado do Ceará, as agregações foram maiores em locais mais baixos e distantes da linha de praia, em que mesmo na maré baixa ainda havia acúmulo de água (ARAÚJO; ROCHA-BARREIRA, 2004), portanto oposto ao ocorrido em Mangue Seco, que teve mais indivíduos no sentido próximo à linha de praia dos estratos ic, im e b2, respectivamente. No Rio de Janeiro, as maiores abundâncias foram também encontradas em local mais próximo à linha de praia (MATTOS; CARDOSO, 2012). No Rio Grande do Norte, foi tomada como referência a distância em relação ao estuário e as maiores abundâncias ocorreram principalmente em locais com proximidade da zona estuarina (BELÉM et al., 2013). Em Mangue Seco também ficou evidente que as zonas próximas a estuários localizadas nos estratos b1 e b4 pareceram mais propícias ao estabelecimento da $A$. flexuosa quando comparadas a locais com maior interferência marinha (b2 e b3).

As densidades médias encontradas no banco de Mangue Seco, de maneira geral, tiveram regiões de altas (ic), intermediárias (b1, b4 e im) e baixas (b2 e b3) densidades. Assim, regiões que tendem a ter altas densidades variaram com média entre 215 e 1013 ind. $\mathrm{m}^{-2}$, as intermediárias entre 106 e 665 ind. $\mathrm{m}^{-2} \mathrm{e}$ as baixas entre 0 e 71 ind. $\mathrm{m}^{-2}$. Densidades maiores em anos anteriores, como em 2006 e 2007, foram encontradas no trabalho de El-Deir et al. (2009) com mínimo de 816 ind. $\mathrm{m}^{-2}$ e máximo de 2016 ind. ind. $\mathrm{m}^{-2}$. No ano de 2009, as densidades estimadas por Oliveira et al. (2011) em Mangue Seco tiveram valores menores quando comparados a este trabalho, com valor mínimo de 103 ind. $\mathrm{m}^{-2} \mathrm{e}$ máximo de 415 ind. $\mathrm{m}^{-2}$. Em outros estados com informações publicadas sobre a A. flexuosa no Brasil, apenas no Rio Grande do Norte (RODRIGUES et al., 2013) foram médias de densidade maiores que neste trabalho, porém se comparadas às densidades estimadas nos anos de 2006 e 2007 para Mangue Seco (EL-DEIR et al., 2009), este banco extrativista foi o que apresentou as maiores médias registradas.

A identificação das características de um local com grande atividade de pesca é importante para a gestão do recurso, pois serve como referencial para o estabelecimento de alternativas de manejo como, por exemplo, áreas de exclusão de pesca, e para isso é necessário o conhecimento dos pontos mais abundantes para que se possa maximizar o impacto da medida nas áreas adjacentes e menos abundantes. $\mathrm{Na}$ divisão do banco de Mangue Seco, de acordo com as características morfodinâmicas e as densidades da A. flexuosa, foi possível diferenciar, principalmente, quatro estratos de localização (ic, im, borda 2 e 3 e borda 1 e 4). O estrato ic foi o mais abundante, portanto o mais propício em caso de adoção dessa medida. No entanto, ressalta-se que o zoneamento deve ser um processo dinâmico que o estabelecimento das áreas neste trabalho serve como ponto inicial de partida para tomadas de decisão e que podem mudar ao longo do tempo, de acordo com novas 
informações da sociedade civil, de pesquisadores e do poder público.

\section{Agradecimentos}

Aos graduandos do curso de Engenharia de Pesca da Universidade Federal Rural de Pernambuco, Ana Júlia Rufino de Freitas e Anderson Thiago Vasconcelos de Freitas, pelo auxílio na obtenção e biometria das amostras. À Coordenação de Aperfeiçoamento de Pessoal de Nível Superior (CAPES), por conceder uma bolsa de estudo de pós-graduação ao primeiro autor.

\section{Referências}

ARAÚJO, M. L. R.; ROCHA-BARREIRA, C. A. Distribuição espacial de Anomalocardia brasiliana (Gmelin, 1791) (Mollusca, Bivalvia, Veneridae) na praia do Canto da Barra, Fortim, Ceará, Brasil. Boletim técnico científico do CEPENE, Tamandaré, v. 12, n. 1, p. 11-21, 2004.

BELÉM, T. P.; MOURA, R. S. T.; HENRY-SILVA, G. G. Distribuição e densidade do bivalve Anomalocardia brasiliana em praias do Rio Grande do Norte durante um período de pluviosidade atípica. Biotemas, Florianópolis, v. 26, n. 1, p. 109-122, 2013.

BOEHS, G.; ABSHER, T. M.; CRUZ-KALED, A. C. Ecologia populacional de Anomalocardia brasiliana (Gmelin, 1791) (Bivalvia, Veneridae) na Baía de Paranaguá, Paraná, Brasil. Boletim do Instituto de Pesca, São Paulo, v. 34, n. 2, p. 259-270, 2008.

CABRAL, A. L.; SASSI, R.; COSTA, C. F. A pesca de subsistência no estuário do rio Timbó, estado de Pernambuco, Brasil. Boletim Técnico Científico do CEPENE, Tamandaré, v. 14, n. 1, p. 111140, 2006.

CORTE, G. N.; YOKOYAMA, L. Q; AMARAL. A. C. Z. An attempt to extend the Habitat Harshness Hypothesis to tidal flats: A case study of Anomalocardia brasiliana (Bivalvia: Veneridae) reproductive biology. Estuarine, Coastal and Shelf Science, London, v. 150, n. 15 p. 136-141, 2014.

CORTE, G. C.; COLEMAN, R. A.; CECÍLIA, A.; AMARAL, Z. Environmental influence on population dynamics of the bivalve Anomalocardia brasiliana. Estuarine, Coastal and Shelf Science, London v. 187, n. 4, p. 241-248, 2017.

EL-DEIR, S.; NEUMANN-LEITÃO, S.; MELO, P. A. M. C. Distribution pattern of Anomalocardia brasiliana Gmelin, 1971 (Mollusca, Bivalvia) in a tropical coastal ecosystem. Tropical Oceanography, Recife, v. 37, n. 1, p. 1-12, 2009.

IBAMA/CEPENE. Boletim estatístico da pesca marítima estuarina do nordeste do Brasil - 2006. Tamandaré, 2008. 385 p. IBAMA. Estatística da pesca 2007 Brasil: grandes regiões e unidades da federação. Brasília: Ibama, 2009. 175 p.

KRUSKAL, W. H.; WALLIS, W. A. Use of ranks in onecriterion variance analysis. Journal of the American Statistical Association, Virginia, v. 47, n. 260, p. 583-621, 1952.
LESSA, R. P.; MONTEIRO, A.; DUARTE-NETO, P. J.; VIEIRA, A. C. Análise multidimensional dos sistemas de produção pesqueira do estado de Pernambuco, Brasil. In: HAIMOVICI, M. (Ed.). Sistemas pesqueiros marinhos e estuarinos do Brasil. Rio Grande: Editora da FURG, 2011. p. 41-54.

MATTOS, G.; CARDOSO, R. S. Population dynamics of two suspension-feeding bivalves on a sheltered beach in southeastern Brazil. Helgoland Marine Research, Bremerhaven, v. 66, n. 3, p. 393-400, 2012.

MOLLUSCABASE. World register of marine species Anomalocardia brasiliana (Gmelin, 1791). 2018. Disponível em: $\quad<$ http://www.marinespecies.org/aphia.php? $\mathrm{p}=$ taxdetails\&id= 420930>. Acesso em: 17 mar. 2018.

MOURA, R. T. Aspectos gerais da hidrobiologia do litoral norte de Pernambuco - Brasil. Brasília: IBAMA, 2009. 138 p.

NARCHI, W. Aspectos ecológicos e adaptativos de alguns bivalves do litoral paulista. Papéis Avulsos de Zoologia, São Paulo, v. 27, n. 19 , p. $235-262,1974$

OLIVEIRA, I. B.; AMORIM, A.; LAVANDER, H.; PEIXOTO, S.; GÁLVEZ, A. O. Spatial and temporal distribution of the shellfish Anomalocardia brasiliana (Gmelin, 1791) on Mangue Seco beach, Pernambuco, Brazil. International Journal of Aquatic Science, Urmia, v. 2, n. 1, p. 68-79, 2011.

OLIVEIRA, I. B.; SILVA NETO, S. R.; LIMA FILHO, J. V. M.; PEIXOTO, S. R. M.; GALVEZ, A. O. Efeito do período chuvoso na extração do molusco bivalve Anomalocardia brasiliana (Gmelin, 1791). Revista Brasileira de Ciências Agrárias, Recife, v. 9, n. 1, p. 139-145, 2014.

PEBESMA, E.; GRAELER, B. Gstat - Spatial and spatio-temporal geostatistical modelling, prediction and simulation. $\mathrm{R}$ package version 1.1-5. Disponível em: <https:/github.com/edzer/gstat/>. Acesso em: 20 dez. 2017.

R CORE TEAM. R - A Language and Environment for Statistical Computing. 2017. Disponível em: $<$ https://www.R-project.org/>. Acesso em: 20 dez. 2017.

RODRIGUES, A. M. L.; BORGES-AZEVEDO, C. M.; COSTA, R. S., HENRY-SILVA, G. G. Population structure of the bivalve Anomalocardia brasiliana, (Gmelin, 1791) in the semi-arid estuarine region of northeastern Brazil. Brazilian Journal of Biology. Rio de Janeiro, v. 73, n. 4, p. 819-833, 2013.

SANTOS, M. R. R., RANIERI, V. E. L. Critérios para análise do zoneamento ambiental como instrumento de planejamento e ordenamento territorial. Ambiente \& Sociedade, São Paulo, v. 16, n. 4, p. 43-62, 2013.

SILVA-CAVALCANTI, J. S.; COSTA, M. Fisheries of Anomalocardia brasiliana in Tropical Estuaries. Pan-American Journal of Aquatic Sciences, Rio Claro, v. 6, n. 2, p. 86-99, 2011. SILVA-CAVALCANTI, J. S.; COSTA, M. F.; ALVES, L. H. B. Seasonal variation in the abundance anddistribution of Anomalocardia flexuosa (Mollusca, Bivalvia, Veneridae) in an estuarine intertidal plain. PeerJ, London, 2018. DOI: 10.7717/ peerj. 4332 . 